\title{
CIC-Erosión: herramienta computacional para la predicción del desgate erosivo en líneas de producción y transporte de hidrocarburos
}

\section{CIC-Erosion: computational tool for predicting erosive wear in transport and production pipelines of hydrocarbons}

\section{CIC-Erosion: ferramenta computacional para previsão do desgate erosivo nas linhas de produção e de transporte de hidrocarbonetos}

\author{
Juan C. Díaz Álvarez*; Alexi Caballero Esteban**; Fernando Corzo Álvarez; Leidy M. Gelves Jerez; \\ Giovanni Juzga León; Christian A. Ariza Quitian.
}

Corporación para la Investigación de la Corrosión, CIC, Piedecuesta, Santander, Colombia.

*jdiaz@corrosion.uis.edu.co **acaballero@corrosion.uis.edu.co

Fecha de recepción: 29 de mayo de 2017

Fecha de aceptación: 10 de septiembre de 2018

\begin{abstract}
Resumen
La presencia de daño mecánico por erosión en la pared de la tubería, daño debido al impacto de partículas sólidas, puede afectar gradualmente la integridad de los componentes que conforman los sistemas de producción y transporte de hidrocarburos. La implementación de métodos o modelos para predecir el desgate erosivo es una actividad de interés para la industria del petróleo, debido a que sus resultados son usados para el estudio, valoración y seguimiento de los procesos erosivos, además de ser considerados en el dimensionamiento de las líneas y determinación de velocidades máximas de flujo, con el fin de mitigar y controlar la erosión. En este sentido, considerando la necesidad de mejorar la implementación de modelos, y análisis e interpretación de resultados de erosión, se presenta en este trabajo una metodología de cálculo, estructurada y de carácter selectivo, para predecir el desgaste erosivo en líneas de producción y transporte de hidrocarburos. Para conducir la metodología hasta un estado operativo práctico, se desarrolló una herramienta computacional (CIC-Erosion), usando el lenguaje de programación C\#. En la fase de desarrollo y aseguramiento de la funcionalidad operativa de la herramienta se realizaron las siguientes subfases: a) definición de la arquitectura del software; b) codificación de los fundamentos teóricos de la metodología de cálculo y de la interfaz del usuario, y c) diagnóstico de la funcionalidad operativa del software usando datos experimentales de tasa de erosión reportados en la literatura e información de casos de estudio hipotéticos. Finalmente, se evaluó el comportamiento predictivo de la metodología de cálculo de erosión usando información de un caso de estudio asociado a un campo de producción de petróleo crudo ubicado en Colombia.
\end{abstract}

En la evaluación de la capacidad de predicción del software se observó que existe concordancia entre los resultados obtenidos con la metodología de cálculo de erosión y la información experimental de campo usada en el estudio. De esta manera, la herramienta computacional CIC-Erosion puede ser considerada una alternativa eficiente y confiable para la predicción del desgaste erosivo en infraestructura usada para la recolección y transporte de hidrocarburos.

Palabras clave: Erosión, transporte de fluidos, herramienta computacional, modelos empíricos, lenguaje de programación C\#. 


\begin{abstract}
The mechanical integrity of pipeline walls in the system components used in hydrocarbons production and transportation can be compromised by the erosion caused by the continuous impact of suspended solids. The implementation of methods or models to monitor and predict erosive wear is an important tool for the oil industry. The results from simulations and prediction models can aid in the calculation and determination of line sizes and maximum flow velocities to control and regulated wall erosion. In this work, we present a structured and selective calculation methodology to improve the implementation of models and the analysis and interpretation of erosion data for the prediction of erosive wear in transport pipelines. A computational tool (ClC-Erosion) written in $\mathrm{C \#}$ programming language was developed to bring the methodology to a practical operational state. To develop and assure the operational functionality of the $\mathrm{CIC}-$ Erosion tool, the following activities were performed: a) definition of software architecture, b) programming of the user interface and theoretical foundations, c) evaluation of the software operational functionality; using experimental erosion rate data reported in the literature and information from hypothetical case studies. Finally, the capacity to predict the behavior of the erosion calculation methodology was assessed using experimental data from case studies identified in the crude oil production of a field located in Colombia. When the predictive capacity of the software was evaluated, a good agreement between results obtained with the erosion calculation methodology and the experimental data was observed. Therefore, the CIC-Erosion computational tool can be considered an efficient and reliable alternative for the prediction of erosive wear in infrastructure used in the hydrocarbon industry.
\end{abstract}

Keywords: Erosion, Fluid Transport, Computational Tool, Empirical Models, C\# Programming Language.

\title{
Resumo
}

A presença de dano mecânico por erosão na parede da tubulação, devido ao impacto das partículas sólidas, pode afetar gradativamente a integridade dos componentes que fazem parte dos sistemas de produção e transporte de hidrocarbonetos. A implementação de métodos ou modelos para prever o desgaste erosivo é uma atividade de interesse para a indústria do petróleo, já que seus resultados são utilizados para o estudo, avaliação e monitoramento de processos erosivos, além de ser considerada no dimensionamento de linhas e na determinação das taxas de fluxo máximo, com o intuito de mitigar e controlar a erosão. Nesse sentido, considerando a necessidade de melhorar a implementação de modelos, a análise e a interpretação dos resultados de erosão, neste trabalho é apresentada uma metodologia de cálculo, estruturada e seletiva, a fim de prever o desgaste erosivo em linhas de produção e de transporte hidrocarbonetos. Para conduzir a metodologia para um estado operacional prático, foi desenvolvido uma ferramenta computacional (ClC-Erosion), utilizando C\# como linguagem de programação. $\mathrm{Na}$ fase de desenvolvimento e garantia de funcionalidade operacional da ferramenta, foram executadas as seguintes etapas: a) definição da arquitetura de software, b) codificação dos fundamentos teóricos da metodologia de cálculo e da interface do usuário, e c) diagnóstico da funcionalidade operacional do software usando dados de taxa de erosão experimentais relatados na informação bibliográfica coletada e informações sobre casos hipotéticos. Finalmente, foi avaliado o desempenho preditivo da metodologia de cálculo da erosão usando informações de um estudo de caso de um campo associado à produção de petróleo localizado na Colômbia.

Na avaliação do poder preditivo do software, observou-se que existe concordância entre os resultados obtidos com a metodologia de cálculo da erosão e os dados experimentais de campo utilizados no estudo. Assim, a ferramenta computacional CIC-Erosion pode ser considerada uma alternativa eficiente e confiável para prever o desgaste erosivo em infraestruturas utilizadas para a produção e transporte de hidrocarbonetos.

Palavras Clave: Erosão, Transporte de fluidos, Ferramenta Computacional, Modelos Empíricos, Linguaje de Programação $C$ \#. 


\section{Introducción}

La presencia de arena en los fluidos de producción es un problema de relevancia para las compañías operadoras de yacimientos de hidrocarburos. Esto se debe a que puede afectar de diversas maneras la rentabilidad del negocio y, en casos críticos, inducir un impacto ambiental por derrames sobre el entorno, causado principalmente por la pérdida de contención del fluido [1].

Dentro de las múltiples problemáticas asociadas a la presencia de arena se destacan: a) obstrucción en los canales de flujo en la formación productora; b) dificultad en la manipulación de grandes volúmenes de sólidos en superficie; b) afectación en los volúmenes de producción por causa de la reducción en el área de flujo asociado a acumulación de sólidos en las tuberías, y finalmente c) erosión por el impacto de partículas sólidas sobre la infraestructura, tanto de fondo de pozo como de superficie. Esta última es una condición considerada como una amenaza para la integridad mecánica de los activos [1,2]. De ahí que sea necesario el planteamiento de estrategias eficaces para la gestión de sólidos, tanto en el manejo y disposición de estos como en los planes de acción para mitigar y controlar la erosión, con lo cual se contribuye al aseguramiento de la integridad mecánica de los activos.

Las compañías operadoras buscan directrices en las fases de diseño y operación que les permitan maximizar las tasas de producción manteniendo la degradación de la tubería por erosión a una tasa permisible. Las directrices actuales estaban basadas, en primera instancia, en restringir la condición de flujo a una velocidad máxima (velocidad límite erosiva), según la recomendación establecida en la norma API RP 14E [3], donde se presenta una ecuación empírica sencilla, para predecir la velocidad límite erosiva en función de una constante $\mathrm{C}$ y la densidad del fluido.

Aunque esta norma ha sido implementada a lo largo de los años, su ecuación no considera muchos factores importantes que afectan el fenómeno de erosión, entre los cuales se destacan: partículas sólidas inmersas en el fluido; el tipo de material de los equipos; las geometrías de los accesorios; las propiedades de los fluidos, y el régimen de flujo, entre otros $[4,5]$. En segundo lugar, algunas compañías han propuesto sus propios criterios de velocidad máxima, según la experiencia de los profesionales en campo. No obstante, este enfoque es insuficiente, debido a que no cuantifica el grado de degradación de la tubería por erosión ocasionado por el impacto de partículas sólidas, lo que oculta la necesidad de formular planes de acción que permitan mitigar y controlar la erosión, prevenir fugas (pérdida de contención del fluido) $y$, con ello, paradas de planta no programadas que resultan muy costosas para la operación. De esta manera, la disponibilidad de metodologías y herramientas computacionales que incorporen nuevos modelos de predicción de erosión en función de múltiples variables permitirá inicialmente que los ingenieros de corrosión e integridad puedan predecir la susceptibilidad a la erosión en las líneas de transporte, y posteriormente establecer la capacidad máxima de producción, para mantener el daño erosivo dentro de límites tolerables, y maximizar el potencial económico de los pozos [5].

Considerando las desventajas que presenta la norma API RP 14E, surgieron algunos modelos empíricos que cuantifican la severidad de la erosión causada por el impacto de partículas sólidas sobre la superficie de la tubería, en términos de pérdida de espesor de pared denotado como tasa de erosión. En este sentido, Salama y Venkatesh [6] propusieron una ecuación para la predicción de tasa de erosión en codos y tees. Este modelo tiene en cuenta la velocidad de flujo, el diámetro de la tubería y la tasa de producción de arena, y fue verificado usando datos de erosión para un sistema de flujo de aire. De esta manera, sus predicciones pueden ser muy precisas principalmente para sistemas de gas. Posteriormente, Salama [4] presentó un modelo aplicable a flujo de dos fases (gas-líquido), considerando el efecto del diámetro de la partícula y la densidad del fluido, y fue validado con datos experimentales obtenidos por diferentes autores. Esta ecuación es una versión mejorada del modelo anterior [6].

Con el fin de ofrecer a la industria un procedimiento de valoración del desgate erosivo y métodos de control y mitigación en sistemas de transporte, la empresa Det Norske Veritas (DNV) desarrolló la práctica recomendada DNV RP 0501 [7]. Este documento presenta ecuaciones empíricas para predecir el desgaste erosivo en flujo de una fase y flujo multifásico en componentes típicos de un sistema de tuberías (tubería recta, codos, tees, reducciones y soldaduras) asociados con producción y transporte de hidrocarburos (aceite, gas) e inyección de agua. En la construcción y validación de los modelos los autores usaron conocimiento empírico del comportamiento de 
la erosión, una ecuación de erosión estándar, información de ensayos experimentales realizados en un circuito de flujo continuo, y resultados de simulación de dinámica de fluidos computacional (CFD, siglas en inglés). A diferencia de los modelos anteriormente mencionados, estas ecuaciones tienen en cuenta el efecto de las propiedades de los materiales de la tubería, el tipo de geometría del accesorio, y propiedades de las partículas sólidas.

Posteriormente, investigadores asociados al Erosion/Corrosion Research Center (E/CRC) de The University of Tulsa desarrollaron un modelo mecanístico exhaustivo formulado para predecir la tasa de erosión en flujo monofásico y multifásico, considerando múltiples variables que afectan el fenómeno de erosión, entre las cuales se destacan: propiedades de los fluidos; propiedades de las partículas sólidas; propiedades de los materiales; características de la geometría de los accesorios, y patrón de flujo [6,8-12]. Posteriormente, los autores llevaron su desarrollo hasta un estado operativo a través de la construcción del software de predicción de erosión SPPS (Sand Production Pipe Saver, por sus siglas en inglés). No obstante, este software tiene disponibilidad restringida, solo puede ser usado por los miembros del E/CRC y algunas compañías (Baker Hughes, Chevron, Halliburton, etc.) que están asociadas al centro de investigación.

Hasta este punto se puede notar el avance que se ha logrado en el modelado del fenómeno de erosión. Sin embargo, es importante mencionar que la multiplicidad de modelos de erosión disponibles en la literatura dificulta su implementación para analizar algún caso de estudio. De otra parte, la aplicación indiscriminada de modelos puede conducir a confusión en la interpretación de los resultados de erosión, ya que se pueden presentar diferencias significativas en la predicción de la tasa de erosión hasta de varios órdenes de magnitud para un mismo caso de estudio.

Debido a las razones anteriormente mencionadas y considerando la necesidad de mejorar la implementación de modelos, y análisis e interpretación de resultados de erosión, en este trabajo se presenta una metodología de cálculo, estructurada y selectiva para predecir el desgaste erosivo en líneas de producción y transporte de hidrocarburos. La metodología está conformada por cuatro tipos de algoritmos, involucrando los modelos de erosión de Salama [4], Salama y Venkatesh [6], DNV RP O501 [7] y E/CRC Tulsa [6,8-12]. Además, la metodología posee un algoritmo de criterios de decisión que le permite implementar el modelo de erosión más adecuado, según las características del sistema en estudio. La metodología fue conducida hasta un nivel operativo a través del desarrollo de una herramienta computacional (CIC-Erosion), usando el lenguaje de programación C\#.

\section{Descripción de la metodología de cálculo de erosión}

La metodología de cálculo de erosión está conformada por cuatro tipos de algoritmos:

- algoritmo de propiedades fisicoquímicas de las fases y de mezcla

- algoritmo de propiedades de flujo

- algoritmo de modelos de predicción de erosión

- algoritmo de criterios de decisión

\section{Algoritmo de propiedades fisicoquímicas de las fases y de mezcla}

Este algoritmo se construyó con el objetivo de calcular las propiedades fisicoquímicas de los fluidos a las condiciones de flujo, entre las cuales se destacan: densidad, viscosidad y tensión superficial. Estas propiedades son necesarias para cálculos posteriores en los algoritmos de propiedades de flujo y predicción de erosión. En la Figura 1, se muestra el diagrama de flujo del algoritmo de cálculo de las propiedades fisicoquímicas de cada una de las fases y de la mezcla (aplica caso multifásico), incorporado en la herramienta software CIC-Erosion. El proceso se inicia con el cálculo de las propiedades de cada una de las fases (gas, agua, aceite), usando como información de entrada las condiciones operacionales y la composición química del fluido. Adicionalmente, se usan reglas de mezcla cuando el fluido tiene una estructura multicomponente. Posteriormente, se calculan las propiedades de la mezcla, las cuales hacen referencia a un parámetro que involucra la fase y la fase líquida. En la construcción del algoritmo se implementaron diversas correlaciones 0 métodos que son ampliamente usados en la industria de los hidrocarburos para la estimación de las propiedades descritas (véase Tabla 1). 


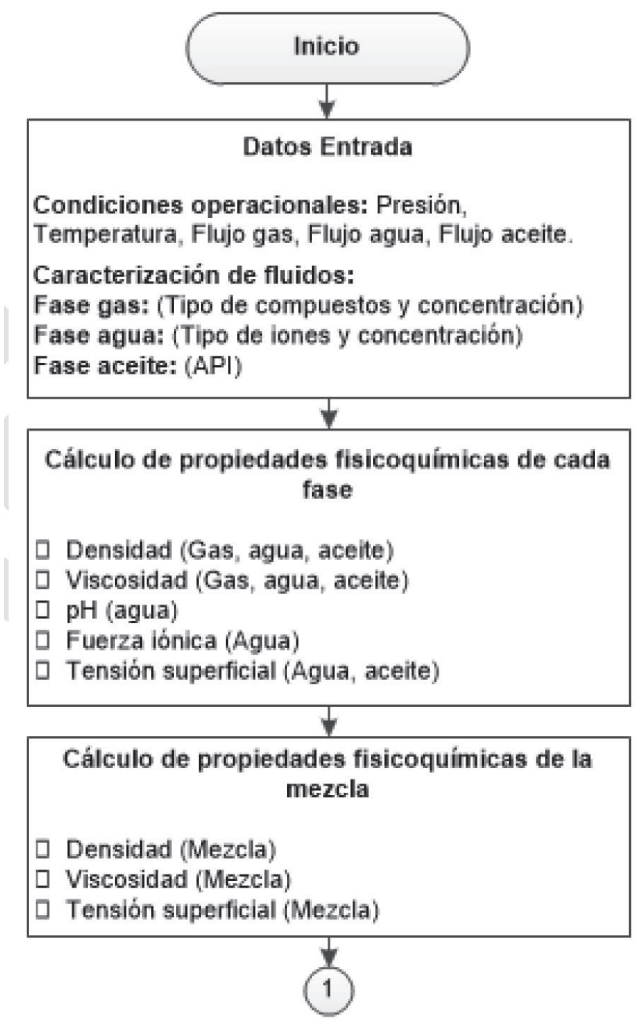

Figura 1. Diagrama de flujo del algoritmo de cálculo de las propiedades fisicoquímicas de las fases y de la mezcla.

Tabla 1. Consolidado de correlaciones para el cálculo de las propiedades fisicoquímicas.

\begin{tabular}{|c|c|c|}
\hline Parámetro & Símbolo & Fuente \\
\hline Solubilidad del gas en el crudo & $\mathrm{R}_{\mathrm{s}}$ & Correlación Glaso [13] \\
\hline Presión punto burbuja & $P_{b}$ & Correlación Glaso [13] \\
\hline $\begin{array}{l}\text { Viscosidad crudo por debajo del punto } \\
\text { de burbuja }\end{array}$ & $\mu_{\mathrm{o}}$ & Correlación Beggs y Robinson [14] \\
\hline $\begin{array}{l}\text { Viscosidad crudo por encima del punto } \\
\text { de burbuja }\end{array}$ & $\mu_{\mathrm{o}}$ & Correlación Beggs y Robinson [14] \\
\hline $\begin{array}{l}\text { Factor volumétrico de formación del } \\
\text { crudo }\end{array}$ & $\mathrm{B}_{0}$ & Correlación Standing [15] \\
\hline $\begin{array}{l}\text { Densidad crudo por debajo del punto } \\
\text { de burbuja }\end{array}$ & $\rho_{\circ}$ & Ahmed [16] \\
\hline $\begin{array}{l}\text { Densidad crudo por encima del punto } \\
\text { de burbuja }\end{array}$ & $\rho_{\circ}$ & Ahmed [16] \\
\hline Tensión superficial crudo & $\sigma_{0}$ & Ghassan y Nimat [17] \\
\hline Viscosidad agua & $\mu_{w}$ & Correlación Meehan [18] \\
\hline Densidad agua & $\rho_{w}$ & API RP 581 Risk-Based Inspection Technology [19] \\
\hline Tensión superficial agua & $\sigma_{w}$ & Sastri y Rao [20] \\
\hline Viscosidad gas & $\mu G$ & Método de Lucas \\
\hline Densidad del gas & $\rho G$ & $\begin{array}{c}\text { Descrito en Poling et al., [21] } \\
\text { Ecuación de estado Peng Robinson Descrita en } \\
\text { Poling et al., [21] }\end{array}$ \\
\hline
\end{tabular}




\section{Algoritmo de propiedades de flujo}

La predicción del desgaste erosivo es una tarea difícil, debido a la existencia de interacciones complejas entre los fluidos y los sólidos suspendidos. La complejidad se intensifica cuando se presenta flujo multifásico, debido a la presencia de patrones de flujo, los cuales afectan de manera diferente el comportamiento de las partículas y, por tanto, la velocidad con la cual impactan la superficie de la tubería. De ahí la importancia de establecer un modelo hidráulico que permita predecir el patrón de flujo y los parámetros hidrodinámicos asociados. Para comprender el comportamiento de flujo multifásico, se implementó el modelo hidráulico propuesto por Taitel y Duckler [22], el cual es un modelo mecanístico exhaustivo formulado para predecir patrones de flujo de dos fases en regiones de transición aplicable a tuberías horizontales y cerca de la horizontal $\left( \pm 15^{\circ}\right)$. Adicionalmente, estima las características de flujo, Holup y gradiente de presión usando la correlación de Lockhart y Martinelli para este último parámetro. Para dar solución al sistema de ecuaciones que describe el modelo hidráulico, se implementó el método numérico de NewtonRaphson, combinado con el método de la secante.
Con respecto a una condición de tubería vertical, se implementó el modelo propuesto por Ansari y Sylvester [23], el cual es también un modelo mecanístico que ha mostrado excelentes resultados en sus predicciones, en comparación con modelos empíricos [24].

Finalmente, para considerar el efecto del patrón de flujo sobre la tasa de erosión, se usaron diversas correlaciones que caracterizan la distribución de las fases (gas, líquid) para cada tipo de régimen de flujo, que posteriormente se relaciona con la velocidad con la cual las partículas de arena golpean la superficie metálica.

En la Figura 2, se muestra el diagrama de flujo del algoritmo de cálculo de las propiedades hidrodinámicas que son usadas en los modelos de predicción de erosión. Una vez definidas las condiciones de operación, diseño y las propiedades fisicoquímicas, el algoritmo inicia con la pregunta ¿el flujo es multifásico? Si la respuesta es negativa, se implementan las ecuaciones convencionales para caracterizar el flujo monofásico; en caso contrario, se implementan los modelos hidráulicos anteriormente mencionados.

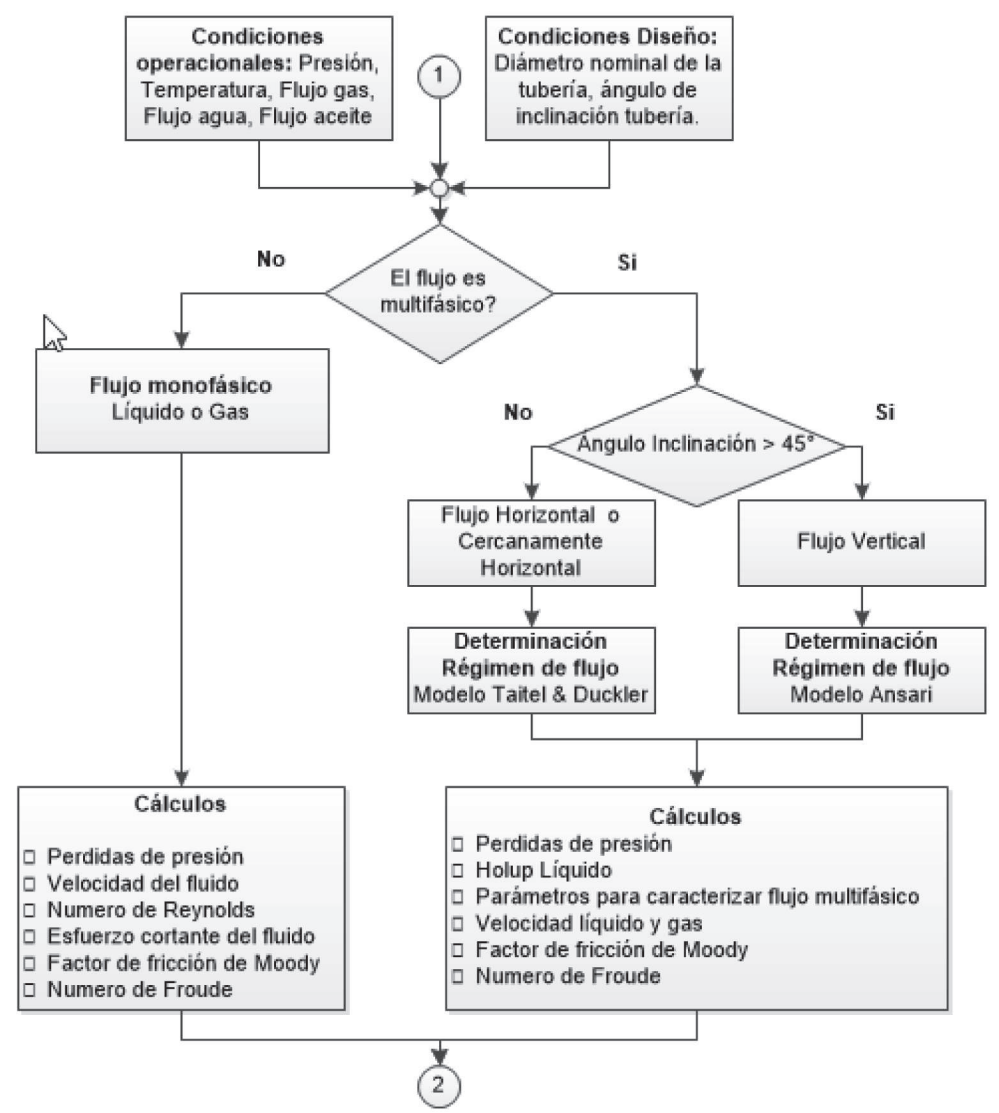

Figura 2. Diagrama de flujo del algoritmo de cálculo de las propiedades hidrodinámicas. 
Algoritmo de modelos de predicción de erosión A través de una revisión del estado del conocimiento en la temática del modelado del fenómeno de erosión, causado por el impacto de partículas sólidas sobre superficies metálicas, se lograron identificar 49 modelos o ecuaciones predictivas $[25,26]$. No obstante, estas ecuaciones generales de predicción de desgaste erosivo tienen aplicabilidad limitada o casi nula en un escenario de campo, debido al tipo de enfoque o concepto científico (teórico y experimental) usado para construir los modelos. Es decir, los modelos están constituidos por parámetros que son imposibles de determinar de manera práctica para una situación en campo o no son fácilmente medibles. Es por esta razón que emergen otros tipos de modelos de amplia aplicación en la industria de los hidrocarburos, entre los cuales se destacan: API RP 14E [3], DNV RP O501 [7], Salama y Venkatesh [6], Salama [4] y E/CRC Tulsa [8-12]. Finalmente, estos modelos son el fundamento teórico del algoritmo para calcular la tasa de erosión o velocidad limite erosiva a través del software CIC-Erosion.

En la Figura 3, se muestra el diagrama de flujo del algoritmo de cálculo del desgaste erosivo, a través de los modelos de erosión. Este algoritmo tiene como información de entrada los resultados obtenidos en los algoritmos de propiedades fisicoquímicas y propiedades de flujo.

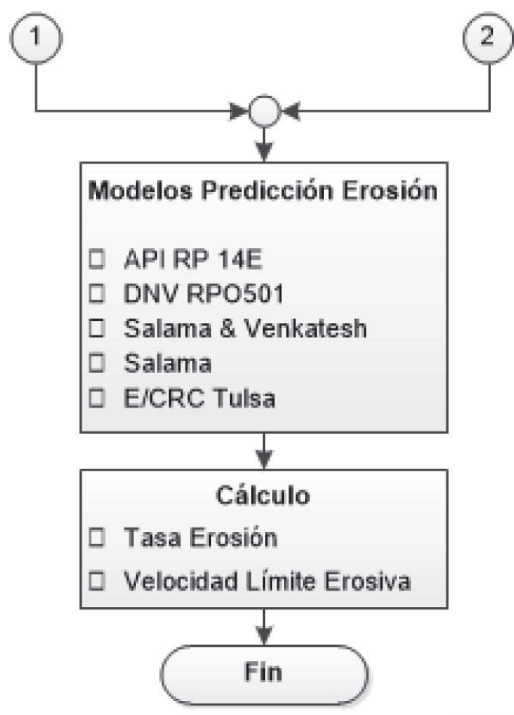

Figura 3. Diagrama de flujo del algoritmo de cálculo del desgaste erosivo.

\begin{abstract}
Algoritmo de criterios de decisión
Este último algoritmo va dirigido a implementar los modelos predictivos más adecuados según las condiciones y características del sistema que se encuentra en estudio. Para cumplir con este objetivo se construyó un algoritmo genérico con criterios de decisión. Dentro de las características necesarias para implementar el algoritmo se destacan: presencia de sólidos (sí o no), tipo de fluido (gas, líquido, multifásico), patrón de flujo (anular, burbuja dispersa, intermitente, slug) y tipo de geometría del accesorio (tramo recto, codo, tee, soldadura, reducción).
\end{abstract}

\section{Desarrollo de la aplicación}

El enfoque general que se utilizó para desarrollar la aplicación software se muestra de manera general en la Figura 4, donde se describe el flujo de información y la interacción informaciónusuario-máquina. Inicialmente, se identifica el sistema que se desea estudiar; pueden ser casos de estudio, tanto en condiciones de laboratorio como en condiciones de campo. En según lugar, el usuario revisa la información, con el fin de garantizar que los datos de los parámetros necesarios para el cálculo de erosión estén disponibles. Posteriormente, existe la interacción 
usuario-máquina, donde el objetivo es ingresar la información en la aplicación, pasar a través de la fase de simulación y obtener los resultados de erosión, los cuales serán recibidos por el usuario.
Finalmente, el usuario analiza los resultados y concluye; en el caso de un sistema de campo, puede definir y ejecutar acciones para mitigar y controlar los fenómenos erosivos.

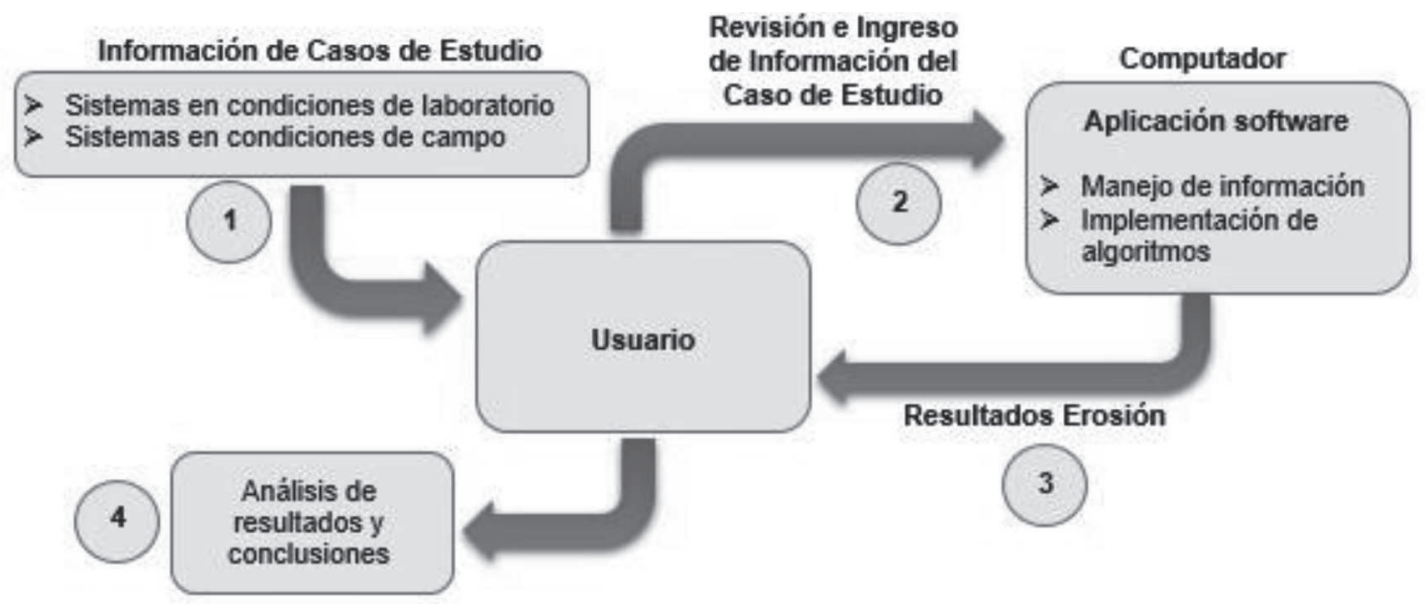

Figura 4. Enfoque general para el desarrollo de la aplicación.

Las directrices o requerimientos de usuario que fueron usados para desarrollar la aplicación son:

- Visualización de la metodología de cálculo: este requerimiento busca que el usuario de la herramienta pueda observar y comprender fácilmente la forma estructural y los criterios usados en la metodología de cálculo para evaluar la susceptibilidad a la erosión.

- Manejo de la información de entrada: el software permite el ingreso de información de un único caso de estudio (ingreso individual de datos sobre la aplicación) y múltiples casos de estudio de manera simultánea (ingreso masivo de datos a través de la importación de un archivo de Excel).

- Manejo de la información de salida: el software consolida y muestra información relevante de la simulación, lo que permite realizar un análisis detallado del o los casos de estudio. Además, tiene la posibilidad de guardar los resultados en diferentes formatos (PDF, Excel) de amplia aplicabilidad y que son fácilmente manipulables para el usuario de la herramienta.

- Especificación de módulos para el ingreso de información: este requerimiento va dirigido a crear módulos de trabajo donde se especifique información de parámetros que están relacionados o que pertenecen a un grupo específico que permite caracterizar el caso de estudio (por ejemplo: condiciones de flujo, propiedades de los fluidos, etc.). Además, considerando que en ciertas situaciones para un caso de estudio en campo no es posible conocer completamente la información de todos los parámetros requeridos, el software tiene la posibilidad de generar información relevante, con el fin de ayudar al usuario a establecer una magnitud veraz y confiable de algún parámetro desconocido, de acuerdo con las características del sistema.

Considerando la estructura anteriormente descrita y los requerimientos de usuario, se desarrolló una aplicación software de escritorio, la cual se describe en la sección de resultados.

\section{Aseguramiento e implementación de la aplicación software}

\section{Diagnóstico de la funcionalidad operativa del software}

En esta fase se realizó un análisis comparativo entre los datos de erosión (velocidad límite erosiva y tasa de erosión) obtenidos con la herramienta software CIC-Erosion, una hoja de cálculo disponible en la web Pipeng Toolbox, e información de cálculos disponibles en la literatura que 
fueron realizados por los autores de los modelos de erosión. Lo anterior se realiza con el fin de garantizar la confiabilidad de los resultados de las operaciones matemáticas incorporadas en los algoritmos de cálculo de la herramienta software CIC-Erosion.

Para realizar esta fase y validar todas las opciones disponibles de tipos de sistemas de transporte de hidrocarburos, se utilizó información de tres casos de estudio hipotéticos generales según el tipo de fluido, especificados en la Tabla 2.

Para validar completamente los procesos matemáticos establecidos en cada una de las rutas de los algoritmos de cálculo se realizaron 74 pruebas, en las cuales se modificó el tipo de fluido (gas, líquido y multifásico), presencia de sólidos (sí, no), tipo de accesorio (tramo recto, codo, tee, tee ciega, soldadura) y forma de la arena (angular, semiredondeada y redondeada). La definición de los parámetros anteriormente mencionados en cada una de las pruebas depende de las características del modelo validado. El número de pruebas realizadas para cada uno de los modelos se muestra en la Tabla 3.

Tabla 2. Información de los casos de estudio hipotéticos.

\begin{tabular}{|c|c|c|c|c|}
\hline $\begin{array}{c}\text { Tipo de } \\
\text { información }\end{array}$ & Parámetro & Caso A & Caso B & Caso C \\
\hline \multirow{8}{*}{$\begin{array}{l}\text { Características de } \\
\text { la infraestructura }\end{array}$} & Diámetro Nominal Tubería (in) & 3 & 3 & 6 \\
\hline & Schedule & Estándar & Estándar & Estándar \\
\hline & Diámetro Interno (m) & 0,0779 & 0,0779 & 0,1540 \\
\hline & Diámetro Interno Pre-reducción (m) & \multicolumn{2}{|c|}{0,0779 (3 in nominal) } & $\begin{array}{l}0,1540(6 \text { in } \\
\text { nominal })\end{array}$ \\
\hline & Diámetro Interno Post-reducción (m) & \multicolumn{2}{|c|}{0,0525 (2 in nominal) } & $\begin{array}{l}0,1022(4 \text { in } \\
\text { Nominal) }\end{array}$ \\
\hline & Tipo de Codo & $\begin{array}{c}\text { Radio Largo } \\
90^{\circ}\end{array}$ & $\begin{array}{c}\text { Radio Largo } \\
90^{\circ}\end{array}$ & Radio Largo $90^{\circ}$ \\
\hline & Radio del Codo (m) & 0,1143 & 0,1143 & 0,2286 \\
\hline & Altura de la Soldadura (m) & $1 \mathrm{E}-3$ & $1 \mathrm{E}-3$ & $2 \mathrm{E}-3$ \\
\hline \multirow{2}{*}{$\begin{array}{l}\text { Parámetros } \\
\text { operacionales }\end{array}$} & Temperatura (K) & 303 & 355 & 344 \\
\hline & Presión (Pa) & $4,14 \mathrm{E} 6$ & $7,06 \mathrm{E} 5$ & 1,38E6 \\
\hline \multirow{3}{*}{$\begin{array}{l}\text { Condiciones de } \\
\text { Flujo }\end{array}$} & Tipo de Fluido o Sistema & Gas & Líquido & Multifásico \\
\hline & Flujo gas (MMSCFD) & 5,09 & 0 & 5,26 \\
\hline & Flujo Líquido (BFPD) & 0 & 7.600 & 18.000 \\
\hline \multirow{4}{*}{$\begin{array}{l}\text { Propiedades del } \\
\text { Fluido }\end{array}$} & Densidad del Gas (kg/m3) & 32,50 & 0 & 22,65 \\
\hline & Densidad del Líquido (kg/m3) & 0 & 955 & 1002,53 \\
\hline & Viscosidad del Líquido (kg/m-s) & 0 & $3,460 \mathrm{E}-04$ & $5,72 \mathrm{E}-04$ \\
\hline & Viscosidad del gas (kg/m-s) & 1,13E-05 & 0 & 1,03E-05 \\
\hline \multirow{4}{*}{$\begin{array}{l}\text { Propiedades de } \\
\text { las partículas } \\
\text { sólidas }\end{array}$} & Diámetro Partícula (m) & $2,80 \mathrm{E}-4$ & $3,07 \mathrm{E}-4$ & $1,85 \mathrm{E}-4$ \\
\hline & Contenido Arena en Gas (Ib/MMSCF) & 10,38 & 0 & 5,02 \\
\hline & Contenido Arena en Líquido (lb/mbbls) & 0 & 55,69 & 8,82 \\
\hline & Densidad Partícula (kg/m3) & 2500 & 2350 & 2200 \\
\hline
\end{tabular}


Tabla 3. Información de número de pruebas realizadas según el modelo predictivo.

\begin{tabular}{|c|c|c|c|}
\hline o. & Modelo & $\begin{array}{l}\text { Número de } \\
\text { Pruebas }\end{array}$ & Observación \\
\hline 1 & API RP 14E & 12 & $\begin{array}{l}\text { Se evalúan los sistemas, líquido y multifásico (casos de estudio } \\
\text { hipotéticos), considerando las diferentes características del } \\
\text { sistema, asociadas a presencia de inhibidor y tipo de servicio } \\
\text { (continuo, intermitente). }\end{array}$ \\
\hline 2 & Salama y Venkatesh & 14 & $\begin{array}{l}\text { Se evalúan } 14 \text { datos de tasa de erosión de un sistema de gas } \\
\text { reportado en la literatura, considerando una geometría de } \\
\text { accesorio tipo codo. }\end{array}$ \\
\hline 3 & Salama & 10 & $\begin{array}{l}\text { Se evalúan } 10 \text { datos de tasa de erosión de un sistema de gas } \\
\text { reportado en la literatura, considerando una geometría de } \\
\text { accesorio tipo codo. }\end{array}$ \\
\hline 4 & DNV RP O501 & 18 & $\begin{array}{l}\text { Se evalúan los sistemas, líquido y multifásico (casos de estudio } \\
\text { hipotéticos) para los diferentes tipos de accesorios (tramo recto, } \\
\text { soldadura aguas arriba, soldadura aguas abajo, codo, tee ciega } \\
\text { y reducción), considerando acero al carbono como material de la } \\
\text { tubería. }\end{array}$ \\
\hline 5 & E/CRC Tulsa & 20 & $\begin{array}{l}\text { Se evalúan } 20 \text { datos de tasa de erosión de un sistema de gas } \\
\text { reportado en la literatura, considerando una geometría de } \\
\text { accesorio tipo codo, material acero al carbono } 1018 \text { y forma de la } \\
\text { arena angular. }\end{array}$ \\
\hline
\end{tabular}

Evaluación de la metodología de cálculo

Para evaluar el comportamiento predictivo de la metodología de cálculo de erosión se usó información asociada a un campo de producción de petróleo crudo ubicado en Colombia. En la Tabla 4 se describe la información de 23 líneas de flujo asociadas a sus respectivos pozos productores pertenecientes al sistema de recolección y transporte de fluidos de producción del campo. Una vez definida la información, se realizó la simulación en el software para cada uno de los sistemas de flujo, se estableció una geometría de accesorio tipo codo y se calculó la velocidad límite erosiva y la tasa de erosión como parámetros relevantes para el análisis.

Tabla 4. Información de los 23 sistemas de flujo asociados a un campo de producción de crudo.

\begin{tabular}{cccccccc}
\hline $\begin{array}{c}\text { No. Línea } \\
\text { de Flujo } \\
\text { de Pozo }\end{array}$ & $\begin{array}{c}\text { Grado de } \\
\text { Material de } \\
\text { la Tubería }\end{array}$ & $\begin{array}{c}\text { Fluido de } \\
\text { Transporte }\end{array}$ & $\begin{array}{c}\text { Diámetro } \\
\text { (in) }\end{array}$ & $\begin{array}{c}\text { Temperatura } \\
\text { (K) }\end{array}$ & $\begin{array}{c}\text { Presión } \\
\mathbf{( P a )}\end{array}$ & $\begin{array}{c}\text { Velocidad } \\
\text { de flujo } \\
\text { (m/s) }\end{array}$ & $\begin{array}{c}\text { Tasa } \\
\text { Producción } \\
\text { Arena } \\
\text { (kg/s) }\end{array}$ \\
\hline CIC-01 & API 5L X60 & Gas-Aceite-Agua & 6 & 369,3 & $7,81 \mathrm{E}+05$ & 2,97 & $6,57 \mathrm{E}-03$ \\
CIC-02 & API 5L X60 & Gas-Aceite-Agua & 3 & 368,7 & $1,93 \mathrm{E}+06$ & 3,91 & $6,17 \mathrm{E}-04$ \\
CIC-03 & API 5L X60 & Gas-Aceite-Agua & 4 & 369,3 & $4,44 \mathrm{E}+05$ & 1,83 & $1,17 \mathrm{E}-03$ \\
CIC-04 & API 5L X60 & Gas-Aceite-Agua & 3 & 327,0 & $1,33 \mathrm{E}+06$ & 4,38 & $2,73 \mathrm{E}-04$ \\
CIC-05 & API 5L X60 & Gas-Aceite-Agua & 4 & 366,5 & $5,27 \mathrm{E}+05$ & 2,41 & $1,80 \mathrm{E}-03$ \\
CIC-06 & API 5L X60 & Gas-Aceite-Agua & 4 & 376,5 & $5,04 \mathrm{E}+05$ & 2,16 & $3,79 \mathrm{E}-04$ \\
CIC-07 & API 5L X60 & Gas-Aceite-Agua & 3 & 358,7 & $1,82 \mathrm{E}+06$ & 2,44 & $2,55 \mathrm{E}-04$ \\
CIC-08 & API 5L X60 & Gas-Aceite-Agua & 3 & 363,2 & $1,81 \mathrm{E}+06$ & 2,97 & $1,04 \mathrm{E}-04$ \\
CIC-09 & API 5L X60 & Gas-Aceite-Agua & 4 & 375,4 & $4,59 \mathrm{E}+06$ & 1,86 & $3,00 \mathrm{E}-04$ \\
CIC-10 & API 5L X60 & Gas-Aceite-Agua & 3 & 369,8 & $1,26 \mathrm{E}+06$ & 2,74 & $4,11 \mathrm{E}-05$ \\
CIC-11 & API 5L X60 & Gas-Aceite-Agua & 3 & 364,8 & $1,82 \mathrm{E}+06$ & 2,77 & $3,08 \mathrm{E}-05$ \\
CIC-12 & API 5L X60 & Gas-Aceite-Agua & 4 & 362,6 & $1,87 \mathrm{E}+06$ & 1,81 & $6,08 \mathrm{E}-05$ \\
CIC-13 & API 5L X60 & Gas-Aceite-Agua & 4 & 370,4 & $4,26 \mathrm{E}+05$ & 0,94 & $3,78 \mathrm{E}-04$ \\
CIC-14 & API 5L X60 & Gas-Aceite-Agua & 4 & 379,3 & $5,30 \mathrm{E}+05$ & 2,73 & $1,67 \mathrm{E}-06$ \\
CIC-15 & API 5L X60 & Gas-Aceite-Agua & 4 & 364,3 & $4,25 \mathrm{E}+05$ & 1,24 & $1,04 \mathrm{E}-04$ \\
CIC-16 & API 5L X60 & Gas-Aceite-Agua & 4 & 369,3 & $4,26 \mathrm{E}+05$ & 1,01 & $6,75 \mathrm{E}-05$ \\
\hline
\end{tabular}


rev.ion. 2018;31(2):7-26. Bucaramanga (Colombia).

\begin{tabular}{cccccccc}
\hline $\begin{array}{c}\text { No. Línea } \\
\text { de Flujo } \\
\text { de Pozo }\end{array}$ & $\begin{array}{c}\text { Grado de } \\
\text { Material de } \\
\text { la Tubería }\end{array}$ & $\begin{array}{c}\text { Fluido de } \\
\text { Transporte }\end{array}$ & $\begin{array}{c}\text { Diámetro } \\
\text { Nominal } \\
(\mathbf{i n})\end{array}$ & $\begin{array}{c}\text { Temperatura } \\
\mathbf{( K )}\end{array}$ & $\begin{array}{c}\text { Presión } \\
\mathbf{( P a )}\end{array}$ & $\begin{array}{c}\text { Velocidad } \\
\text { de flujo } \\
\mathbf{( m / s )}\end{array}$ & $\begin{array}{c}\text { Tasa } \\
\text { Producción } \\
\text { Arena } \\
(\mathbf{k g} / \mathbf{s})\end{array}$ \\
\hline CIC-17 & API 5L X60 & Gas-Aceite-Agua & 4 & 366,5 & $1,73 \mathrm{E}+06$ & 3,54 & $2,78 \mathrm{E}-07$ \\
CIC-18 & API 5L X60 & Gas-Aceite-Agua & 3 & 372,0 & $1,57 \mathrm{E}+06$ & 0,54 & $3,53 \mathrm{E}-05$ \\
CIC-19 & API 5L X60 & Gas-Aceite-Agua & 4 & 363,2 & $9,21 \mathrm{E}+05$ & 1,02 & $5,28 \mathrm{E}-06$ \\
CIC-20 & API 5L X60 & Gas-Aceite-Agua & 4 & 373,2 & $8,40 \mathrm{E}+05$ & 2,28 & $4,42 \mathrm{E}-07$ \\
CIC-21 & API 5L X60 & Gas-Aceite-Agua & 4 & 367,6 & $4,62 \mathrm{E}+05$ & 1,46 & $1,11 \mathrm{E}-06$ \\
CIC-22 & API 5L X60 & Gas-Aceite-Agua & 6 & 336,5 & $5,43 \mathrm{E}+05$ & 0,17 & $3,98 \mathrm{E}-06$ \\
CIC-23 & API 5L X60 & Gas-Aceite-Agua & 4 & 361,5 & $8,80 \mathrm{E}+05$ & 1,32 & $5,56 \mathrm{E}-10$ \\
\hline
\end{tabular}

\section{Resultados y discusión}

\section{Descripción de la aplicación software}

Generalidades. Con el fin de ofrecer una aplicación de fácil uso en la implementación de la metodología de cálculo de erosión, se desarrolló una herramienta software de escritorio (ClCErosion), la cual fue diseñada para ser ejecutada en equipos de cómputo con sistemas operativos Windows 7, 8 o 10. Para esto se utilizó el lenguaje de programación C\# y librerías de Net Framework, tanto para la codificación de la lógica de usuario como para los algoritmos de valoración del desgaste erosivo. De igual manera, se crearon las interfaces de usuario, con el fin de ofrecer una experiencia de navegación clara y natural para el usuario del software.

En la Figura 5, se muestra la imagen de bienvenida al software CIC-Erosion, la cual emerge al iniciar la aplicación mientras se carga la visualización de las ventanas para el usuario. La pantalla inicial de CIC-Erosion muestra un diagrama de flujo de la metodología global utilizada para los cálculos de erosión, donde se muestra de una forma didáctica el proceso del cálculo (véase Figura 6). La ventana tiene unos botones en la parte superior que permiten ampliar, reducir, girar y mover la imagen en forma de vista preliminar.

En la barra de menú ubicada en la parte superior de la pantalla principal se describen las opciones que dirigen el modo de navegación en la herramienta (archivo, entrada de datos, resultados), lo que permite que el usuario realice fácilmente la fase de simulación. Se inicia en la opción entrada de datos, posteriormente realiza la simulación en la opción resultados, y finalmente el almacenamiento de la información en la opción archivo (véase Figura 7). Además, en la barra de menú se muestran las opciones, manual de usuario, salir y herramientas. En la opción manual de usuario se puede visualizar un documento donde se describen ciertos detalles de la herramienta, iniciando desde los prerrequisitos técnicos del sistema para instalar la herramienta software, hasta una descripción de las diferentes operaciones que se pueden realizar en términos de cálculo de erosión. En el botón herramientas se tiene una opción llamada conversor, la cual es muy útil para el usuario, ya que permite realizar la conversión de unidades de diferentes magnitudes físicas y químicas. Finalmente, la opción salir permite terminar cualquier operación y cerrar la aplicación.

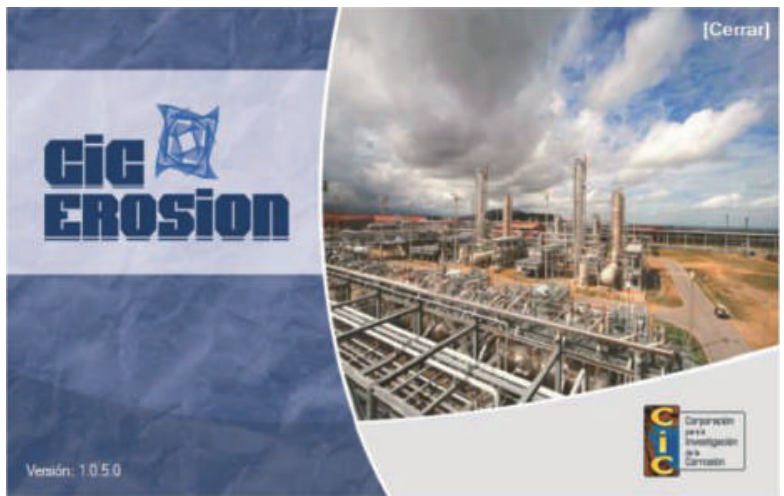

Figura 5. Imagen de bienvenida cuando se inicia la aplicación 


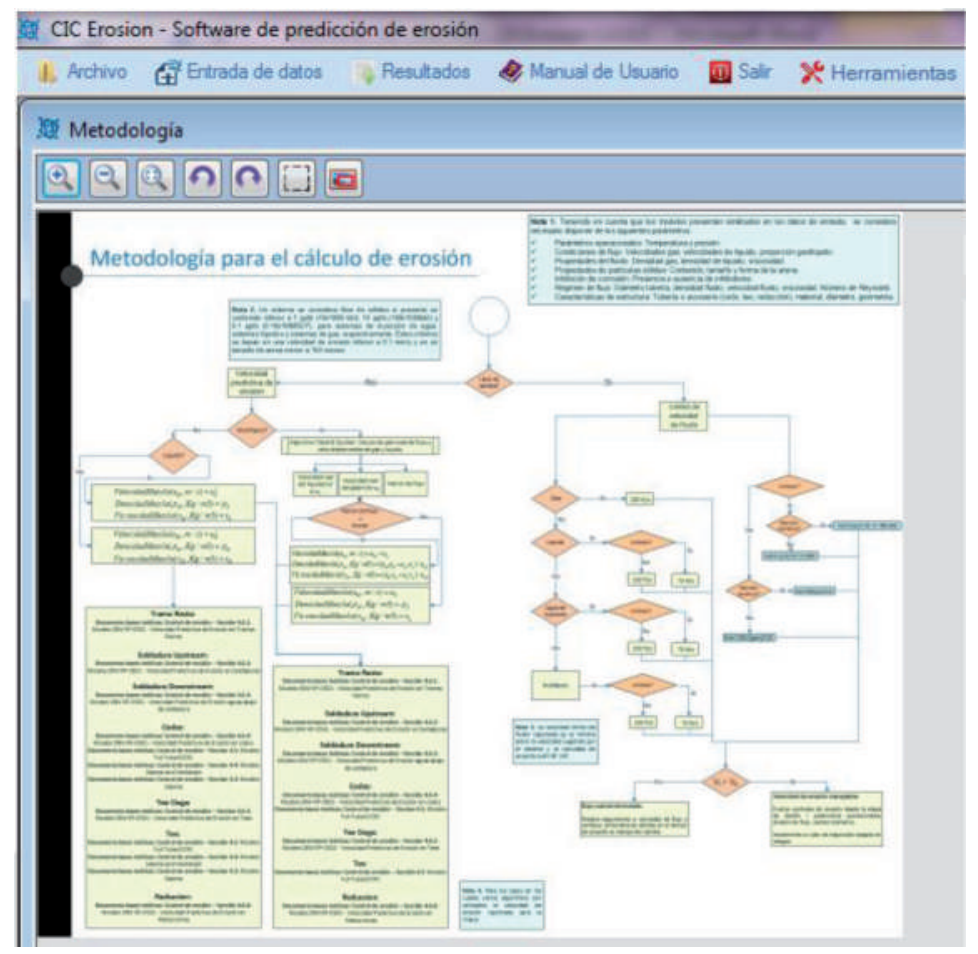

Figura 6. Imagen de la pantalla principal del software CIC-Erosion.

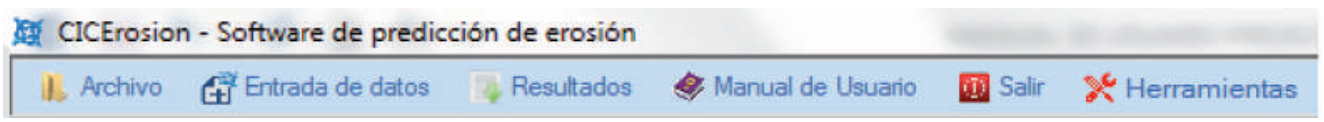

Figura 7. Imagen de la barra de menú principal.

\section{Descripción de las opciones de menú}

Archivo. El manejo de archivos en CIC-Erosion permite guardar la información ingresada de datos de corrientes y la sesión en general. Utiliza archivos con extensión prdero., que corresponden a sesiones de simulación de CIC-Erosion. Esto permite que los datos de las corrientes puedan ser transferibles mediante sus archivos de sesión de simulación.

Entrada de información. El ingreso de la información se realiza a través de las dos opciones que CICErosion presenta: ingreso de información de corrientes (una única corriente) e importación masiva de datos (mediante plantilla de Excel). En las Figuras 8,9 y 10, se muestra la ventana para el ingreso de datos de una única corriente. En el caso de ingreso masivo de datos el software genera una plantilla en Excel, la cual puede ser usada para ingresar información de varias corrientes (límite máximo, 1000 corrientes de flujo) (véase Figura 11). Acontinuación, se describe la información necesaria para simular el sistema que se desea estudiar.

\section{Datos de entrada}

- Información de la infraestructura: geometría del accesorio, material, diámetro.

- Información del sistema: tipo de fluido (gas, líquido, multifásico), presencia de inhibidor (Sí o No), tipo de servicio (continuo o intermitente), sólidos presentes (Sí o No).

- Datos operacionales: presión, temperatura, flujo gas, flujo de líquido, y flujo de producción de arena.

- Propiedades de los fluidos: densidad y viscosidad del gas y líquido.

- Propiedades de las partículas: densidad, diámetro, y forma de la arena.

Salida de información. Los resultados de predicción de erosión se muestran en tablas, discretizados como corrientes con sólidos o sin sólidos (véase Figura 12). A continuación, se describe el tipo de información de salida que se genera al simular el sistema en estudio. 


\section{Datos de salida}

- Nombre de la corriente.

- Variables del sistema (geometría del accesorio, material, tipo de fluido y velocidad de flujo).
- Velocidad límite erosiva o tasa de erosión considerando diferentes modelos predictivos.

- Comentarios y recomendaciones generales.

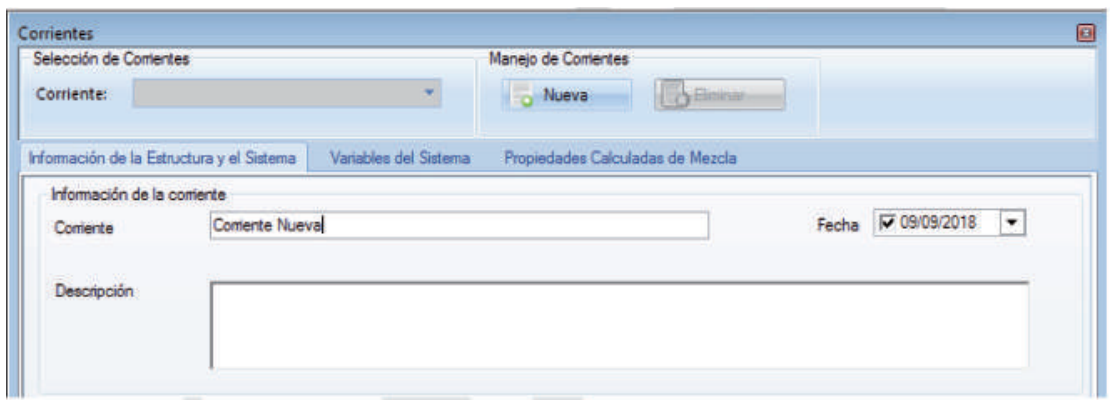

Figura 8. Imagen del módulo de entrada de datos, sección A.

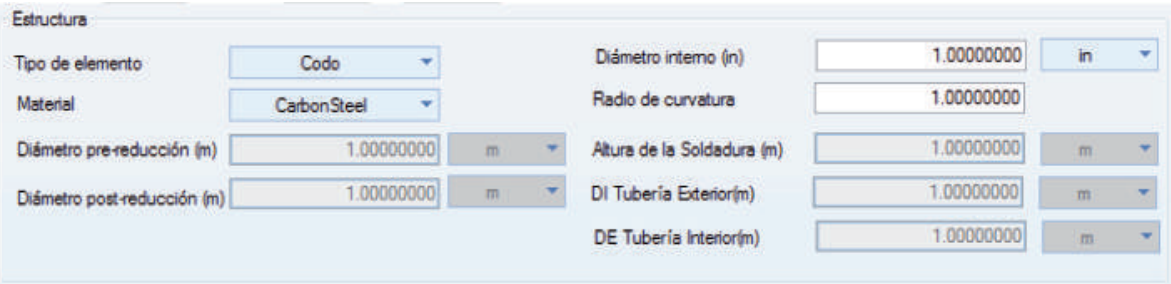

Figura 9. Imagen del módulo de entrada de datos, sección B.

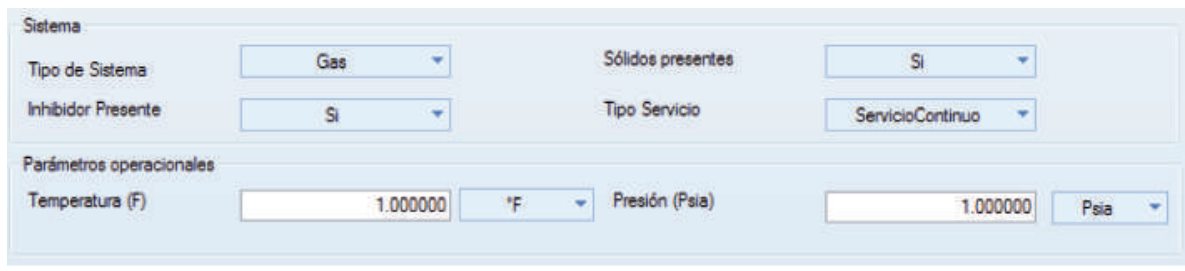

Figura 10. Imagen del módulo de entrada de datos, sección C.

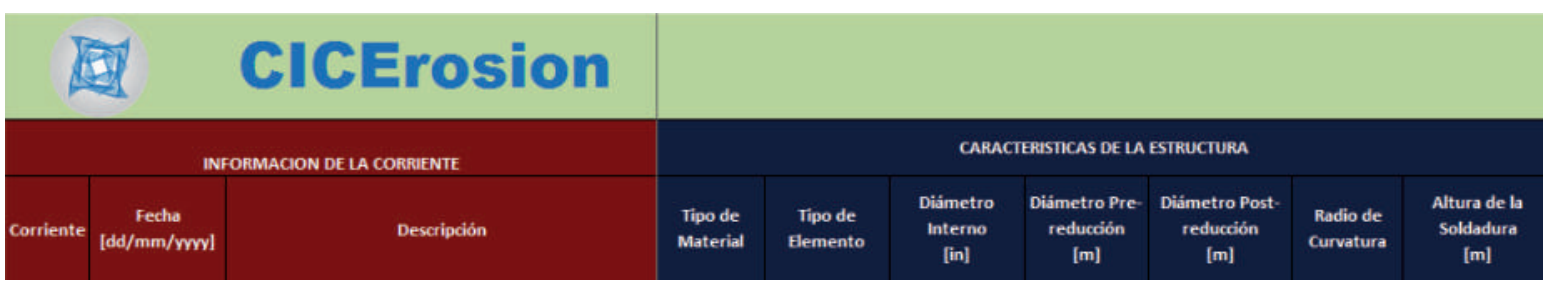

Figura 11. Imagen de la plantilla para ingreso masivo de datos, parte A.

\begin{tabular}{|c|c|c|c|c|c|c|c|c|}
\hline \multicolumn{9}{|c|}{ Resultados corrientes con sólidos } \\
\hline \multicolumn{9}{|c|}{ Resultados con solidos: 1 ltems } \\
\hline Nombre Corriente & Descripción & $\begin{array}{c}\text { Fecha } \\
\text { (dd-mm-yyyy) }\end{array}$ & Tipo Estructura & Material & Sistema & $\begin{array}{c}\text { Velocidad Mezcla } \\
\text { (ft/s) }\end{array}$ & $\begin{array}{c}\text { Tasa de Erosión DNV } \\
\text { (mpy) }\end{array}$ & Tasa de Erosión Aceptable \\
\hline Corriente Nueva & & $17-11 \cdot 2015$ & Tuberia & CarbonSteel & Multifásico & 7.066 & 4.69E-005 & \\
\hline
\end{tabular}

Figura 12. Imagen del módulo de salida de resultados. 
Funcionamiento operativo (comparación datos de simulación)

En esta sección se presenta un análisis comparativo de datos de erosión (velocidad límite erosiva y tasa de erosión) obtenidos con la herramienta software CIC-Erosion, una hoja de cálculo disponible en la web Pipeng Toolbox, e información de cálculos disponibles en la literatura que fueron realizados por los autores de los modelos de erosión.

Modelo API RP 14E. Para este modelo se evaluaron los tres casos de estudio hipotéticos descritos en la metodología, considerando las diferentes características del sistema, asociadas a presencia de inhibidor y tipo de servicio (continuo, intermitente), lo cual corresponde a 12 pruebas.

En la Tabla 5, se muestran los datos de predicción de velocidad límite erosiva obtenidos con el software CIC-Erosion y la hoja de cálculo Pipeng Toolbox: Módulo API RP 14E. Se observa que los datos obtenidos con el software CIC-Erosion son muy similares a los obtenidos en la hoja de cálculo, mostrando porcentajes de error o desviación inferiores a 1,6E-3 \%. En consecuencia, se puede afirmar que las operaciones matemáticas que fueron incorporadas en el software CIC-Erosion son confiables, y corresponden a lo descrito en la norma recomendada API RP 14E.

Tabla 5. Comparación de datos de velocidad límite erosiva obtenidos con la hoja de cálculo Pipeng Toolbox: Módulo API RP 14E y el software CIC-Erosion para el modelo API RP 14E

\begin{tabular}{|c|c|c|c|c|c|}
\hline \multirow[b]{2}{*}{$\begin{array}{l}\text { Caso de } \\
\text { Estudio }\end{array}$} & \multirow{2}{*}{\multicolumn{2}{|c|}{ Características del Sistema }} & \multicolumn{2}{|c|}{ Velocidad Límite Erosiva (m/s) } & \multirow[b]{2}{*}{ \% Error } \\
\hline & & & $\begin{array}{l}\text { Hoja de cálculo } \\
\text { "Pipeng Toolbox }\end{array}$ & $\begin{array}{l}\text { Software CIC- } \\
\text { Erosion }\end{array}$ & \\
\hline \multirow{4}{*}{ A (Gas) } & \multirow{2}{*}{$\begin{array}{l}\text { Sistema con } \\
\text { Inhibidor }\end{array}$} & Servicio Continuo & 32,1 & 32,1 & 3,91E-04 \\
\hline & & Servicio Intermitente & 53,5 & 53,5 & 2,01E-04 \\
\hline & \multirow{2}{*}{$\begin{array}{l}\text { Sistema sin } \\
\text { Inhibidor }\end{array}$} & Servicio Continuo & 21,4 & 21,4 & $8,43 E-05$ \\
\hline & & Servicio Intermitente & 26,7 & 26,7 & 2,01E-04 \\
\hline \multirow{4}{*}{ B (Líquido) } & \multirow{2}{*}{$\begin{array}{l}\text { Sistema con } \\
\text { Inhibidor }\end{array}$} & Servicio Continuo & 5,9 & 5,9 & 1,32E-03 \\
\hline & & Servicio Intermitente & 9,9 & 9,9 & 2,93E-04 \\
\hline & \multirow{2}{*}{$\begin{array}{l}\text { Sistema sin } \\
\text { Inhibidor }\end{array}$} & Servicio Continuo & 3,9 & 3,9 & $1,25 \mathrm{E}-03$ \\
\hline & & Servicio Intermitente & 4,9 & 4,9 & 2,93E-04 \\
\hline \multirow{4}{*}{ C (Multifásico) } & \multirow{2}{*}{$\begin{array}{l}\text { Sistema con } \\
\text { Inhibidor }\end{array}$} & Servicio Continuo & 13,2 & 13,2 & 7,19E-04 \\
\hline & & Servicio Intermitente & 22,0 & 22,0 & 2,03E-04 \\
\hline & \multirow{2}{*}{$\begin{array}{l}\text { Sistema sin } \\
\text { Inhibidor }\end{array}$} & Servicio Continuo & 8,8 & 8,8 & $1,59 \mathrm{E}-03$ \\
\hline & & Servicio Intermitente & 11,0 & 11,0 & $1,18 \mathrm{E}-03$ \\
\hline
\end{tabular}

Modelo Salama y Venkatesh. En la Tabla 6, se muestran los datos de predicción de tasa de erosión reportados por Salama y Venkatesh [6] y obtenidos con el software CIC-Erosion para 14 datos experimentales disponibles en la literatura. Se observa que los datos obtenidos con el software CIC-Erosion son similares a los obtenidos con la hoja de cálculo, mostrando porcentajes de error o desviaciones de 0,25\%. Lo anterior confirma que el algoritmo de cálculo del modelo Salama y Venkatesh es confiable, veraz, y sus resultados están en concordancia con la información reportada en la literatura. 
rev.ion. 2018;31(2):7-26. Bucaramanga (Colombia).

Tabla 6. Comparación de datos de tasa de erosión reportados en la literatura y obtenidos con el software CIC-Erosion para el modelo Salama y Venkatesh considerando flujo de una fase (gas) y geometría del accesorio tipo codo

\begin{tabular}{ccccccc}
\hline No. & $\begin{array}{c}\text { Velocidad del } \\
\text { Gas (ft/s) }\end{array}$ & $\begin{array}{c}\text { Flujo Arena } \\
\text { (bbl/mes) }\end{array}$ & $\begin{array}{c}\text { Dato } \\
\text { Experimental }\end{array}$ & $\begin{array}{c}\text { Tasa Erosión (in/y) } \\
\text { Dato reportado por }\end{array}$ & $\begin{array}{c}\text { Software } \\
\text { Salama Venkatesh (A) } \\
\text { CIC-Erosion }\end{array}$ & \\
\hline 1 & 50 & 143,9 & 71,13 & 107,93 & 107,66 & 0,25 \\
2 & 70 & 133,66 & 193,41 & 196,48 & 195,99 & 0,25 \\
3 & 70 & 144,37 & 206,27 & 212,22 & 211,69 & 0,25 \\
4 & 70 & 144,37 & 202,37 & 212,22 & 211,69 & 0,25 \\
5 & 70 & 86,62 & 102,43 & 127,33 & 127,01 & 0,25 \\
6 & 70 & 86,62 & 86,64 & 127,33 & 127,01 & 0,25 \\
7 & 70 & 86,62 & 84,81 & 127,33 & 127,01 & 0,25 \\
8 & 70 & 113,63 & 77,2 & 167,04 & 166,62 & 0,25 \\
9 & 70 & 113,63 & 78,84 & 167,04 & 166,62 & 0,25 \\
10 & 70 & 113,63 & 91,35 & 167,04 & 166,62 & 0,25 \\
11 & 100 & 120,15 & 253,04 & 360,45 & 359,55 & 0,25 \\
12 & 100 & 120,15 & 257,24 & 360,45 & 359,55 & 0,25 \\
13 & 100 & 120,15 & 296,63 & 360,45 & 359,55 & 0,25 \\
14 & 100 & 141,57 & 555,43 & 424,71 & 423,65 & 0,25 \\
\hline
\end{tabular}

Notas: (A) Estos datos corresponden a la predicción realizada por Salama y Venkatesh [6].

Modelo Salama. Para este modelo se realizaron 10 simulaciones con el software CIC-Erosion correspondientes a datos experimentales reportados en la literatura referidos a un sistema de gas (Salama, [4]).

En la Tabla 7, se muestran los datos de predicción de tasa de erosión reportados por Salama [4] y obtenidos con el software CIC-Erosion para 10 datos experimentales disponibles en la literatura. Se observa que los datos obtenidos con el software CIC-Erosion son muy similares a los reportados, mostrando porcentajes de error o desviaciones inferiores a $0,45 \%$. Lo anterior confirma que los resultados obtenidos con el software CIC-Erosion para el modelo Salama son altamente confiables según la literatura científica.

Tabla 7. Comparación datos de tasa de erosión reportados en la literatura y obtenidos con el software CIC-Erosion para el modelo Salama considerando flujo de una fase (Gas) y geometría del accesorio tipo codo

\begin{tabular}{|c|c|c|c|c|c|c|c|c|}
\hline \multirow[b]{2}{*}{ No. } & \multirow[b]{2}{*}{$\begin{array}{c}\text { Velocidad } \\
\text { Superficial } \\
\text { gas }(\mathrm{m} / \mathrm{s})\end{array}$} & \multirow[b]{2}{*}{$\begin{array}{c}\text { Diámetro } \\
\text { Partícula } \\
\text { (Micrones) }\end{array}$} & \multirow[b]{2}{*}{$\begin{array}{l}\text { Densidad } \\
\text { del Gas } \\
(\mathrm{kg} / \mathrm{m} 3)\end{array}$} & \multirow[b]{2}{*}{$\begin{array}{l}\text { Diámetro } \\
\text { del Codo } \\
(\mathrm{mm})\end{array}$} & \multicolumn{3}{|c|}{ Tasa Erosión $(\mathrm{mm} / \mathrm{kg})$} & \multirow[b]{2}{*}{$\begin{array}{c}\% \\
\text { Error }\end{array}$} \\
\hline & & & & & $\begin{array}{c}\text { Dato } \\
\text { Experimental }\end{array}$ & $\begin{array}{c}\text { Dato } \\
\text { reportado por } \\
\text { Salama }^{(\mathrm{A})}\end{array}$ & $\begin{array}{c}\text { Software } \\
\text { CIC-Erosion }\end{array}$ & \\
\hline 1 & 9,15 & 300 & 1,2 & 52,5 & 2,14E-03 & 3,80E-03 & 3,797E-03 & 0,08 \\
\hline 2 & 12,2 & 300 & 1,2 & 52,5 & $3,81 \mathrm{E}-03$ & $6,75 \mathrm{E}-03$ & $6,750 \mathrm{E}-03$ & 0,00 \\
\hline 3 & 15,25 & 300 & 1,2 & 52,5 & $7,52 \mathrm{E}-03$ & 1,05E-02 & $1,055 \mathrm{E}-02$ & 0,45 \\
\hline 4 & 18,3 & 300 & 1,2 & 52,5 & $9,16 \mathrm{E}-03$ & 1,52E-02 & 1,519E-02 & 0,08 \\
\hline 5 & 21,35 & 300 & 1,2 & 52,5 & $1,22 \mathrm{E}-02$ & 2,07E-02 & 2,067E-02 & 0,13 \\
\hline 6 & 24,4 & 300 & 1,2 & 52,5 & 1,62E-02 & 2,70E-02 & $2,700 \mathrm{E}-02$ & 0,00 \\
\hline 7 & 27,45 & 300 & 1,2 & 52,5 & 1,80E-02 & $3,42 \mathrm{E}-02$ & 3,417E-02 & 0,08 \\
\hline 8 & 30,5 & 300 & 1,2 & 52,5 & 2,04E-02 & 4,22E-02 & $4,219 \mathrm{E}-02$ & 0,03 \\
\hline 9 & 21,35 & 300 & 1,2 & 52,5 & 4,44E-03 & 2,07E-02 & 2,067E-02 & 0,13 \\
\hline 10 & 30,5 & 300 & 1,2 & 52,5 & 1,56E-02 & $4,22 \mathrm{E}-02$ & 4,219E-02 & 0,03 \\
\hline
\end{tabular}

Notas: (A) Estos datos corresponden a la predicción realizada por Salama [4]. 
Modelo DNV RP0501. Para este modelo se evaluaron los tres casos de estudio hipotéticos descritos en la metodología, considerando los tipos de geometría (tramo recto, soldadura, codo, tee ciega y reducción) y acero al carbono como material de la tubería, lo cual corresponde a 18 pruebas.

En la Tabla 8, se muestra el consolidado de los datos de predicción de tasa de erosión obtenidos con el software CIC-Erosion y la hoja de cálculo Pipeng Toolbox: Módulo DNV RP 0501 para los casos de estudio considerados. Al observar los resultados, se puede afirmar que, de las 18 simulaciones realizadas, 13 presentan porcentajes de error inferiores al 0,8 \%, y 5 presentan porcentajes de error entre $1,2 \%$ y $4,6 \%$. La presencia de diferencias relativamente moderadas $(1,2-4,6 \%)$ se debe a que la hoja de cálculo Pipeng Toolbox: Módulo DNV RP 0501 no permite el ingreso del valor de la densidad del gas para los sistemas gas y multifásico, realizando su propio cálculo interno, lo cual genera un impacto directo sobre las ecuaciones matemáticas que depende de este parámetro, y posteriormente sobre la predicción de la tasa de erosión. Estos resultados demuestran que el algoritmo de cálculo implementado en el software CIC-Erosion es completamente veraz y confiable, y corresponde a lo que está documentado en la práctica recomendada DNV RP O501 Erosive Wear in Piping Systems.

Tabla 8. Comparación datos de tasa de erosión obtenidos con la hoja de cálculo Pipeng Toolbox: Módulo DNV RP O501, y software CIC-Erosion para el modelo DNV RP O501

\begin{tabular}{|c|c|c|c|c|}
\hline \multirow[b]{2}{*}{$\begin{array}{l}\text { Caso de } \\
\text { Estudio }\end{array}$} & \multirow[b]{2}{*}{ Geometría del Accesorio } & \multicolumn{2}{|c|}{ Tasa Erosión (mpy) } & \multirow[b]{2}{*}{$\%$ Error } \\
\hline & & $\begin{array}{l}\text { Hoja de cálculo "Pipeng } \\
\text { Toolbox: Módulo DNV RP } \\
\text { O501" }\end{array}$ & $\begin{array}{l}\text { Software CIC- } \\
\text { Erosion }\end{array}$ & \\
\hline \multirow{6}{*}{ A (Gas) } & Tramo Recto & 0,01360 & 0,01359 & 0,093 \\
\hline & Soldadura aguas arriba & 4,33147 & 4,36114 & 0,685 \\
\hline & Soldadura aguas abajo & 0,03142 & 0,03139 & 0,096 \\
\hline & Codo & 7,00746 & 7,00733 & 0,002 \\
\hline & Tee Ciega & 5,54458 & 5,29299 & 4,538 \\
\hline & Reducción & 33,76857 & 33,99991 & 0,685 \\
\hline \multirow{6}{*}{ B (Líquido) } & Tramo Recto & 0,00590 & 0,00590 & 0,005 \\
\hline & Soldadura aguas arriba & 0,62252 & 0,62742 & 0,787 \\
\hline & Soldadura aguas abajo & 0,01364 & 0,01364 & 0,003 \\
\hline & Codo & 0,48242 & 0,48243 & 0,004 \\
\hline & Tee Ciega & 0,12457 & 0,12457 & 0,004 \\
\hline & Reducción & 4,85286 & 4,89146 & 0,795 \\
\hline \multirow{6}{*}{ C (Multifásico) } & Tramo Recto & 0,01557 & 0,01554 & 0,172 \\
\hline & Soldadura aguas arriba & 2,20832 & 2,18089 & 1,242 \\
\hline & Soldadura aguas abajo & 0,05653 & 0,05643 & 0,172 \\
\hline & Codo & 1,77506 & 1,72197 & 2,991 \\
\hline & Tee Ciega & 0,46920 & 0,45489 & 3,049 \\
\hline & Reducción & 18,59258 & 18,36589 & 1,219 \\
\hline
\end{tabular}


Modelo E/CRC Tulsa. Para este modelo se realizaron veinte simulaciones con el software CICErosion correspondientes a datos experimentales reportados en la literatura referidos a un sistema de gas (McLaury et al., [5]).

En la Tabla 9, se muestran los datos de predicción de tasa de erosión reportados por McLaury et al., [5] y obtenidos con el software CIC-Erosion para los veinte datos experimentales disponibles en la literatura. Se observa que los datos obtenidos con el software CIC-Erosion son casi idénticos a los reportados por McLaury et al., [5], mostrando porcentajes de error o desviaciones inferiores al $1,52 \%$. Estos resultados reafirman que las ecuaciones matemáticas y las operaciones numéricas que conforman el algoritmo de cálculo del modelo E/CRC Tulsa implementado en el software CIC-Erosion son totalmente veraces y corresponden a lo desarrollado y reportado por el Erosion/Corrosion Research Center (E/CRC) de The University of Tulsa.

Tabla 9. Comparación de datos de tasa de erosión reportados en la literatura y obtenidos con el software CIC-

Erosion para el modelo E/CRC Tulsa considerando flujo de una fase (as)

\begin{tabular}{|c|c|c|c|c|c|c|c|}
\hline \multirow[b]{2}{*}{ No. } & \multirow[b]{2}{*}{$\begin{array}{l}\text { Velocidad } \\
\text { Superficial } \\
\text { gas }(\mathrm{m} / \mathrm{s})\end{array}$} & \multirow[b]{2}{*}{$\begin{array}{l}\text { Diámetro } \\
\text { Partícula } \\
\text { (Micrones) }\end{array}$} & \multirow[b]{2}{*}{$\begin{array}{l}\text { Radio } \\
\text { Curvatura }\end{array}$} & \multicolumn{3}{|c|}{ Tasa Erosión (mm/kg) } & \multirow[b]{2}{*}{$\%$ Error } \\
\hline & & & & $\begin{array}{c}\text { Dato } \\
\text { Experimental }\end{array}$ & $\begin{array}{c}\text { Dato reportado } \\
\text { por McLaury et } \\
\text { al., [5] }\end{array}$ & $\begin{array}{c}\text { Software } \\
\text { CIC-Erosion }\end{array}$ & \\
\hline 1 & 9,15 & 300 & 1,5 & $2,14 \mathrm{E}-03$ & $2,66 \mathrm{E}-03$ & $2,62 \mathrm{E}-03$ & 1,34 \\
\hline 2 & 12,2 & 300 & 1,5 & 3,81E-03 & 4,38E-03 & $4,33 \mathrm{E}-03$ & 1,22 \\
\hline 3 & 15,25 & 300 & 1,5 & $7,52 \mathrm{E}-03$ & $6,45 \mathrm{E}-03$ & $6,37 \mathrm{E}-03$ & 1,19 \\
\hline 4 & 18,3 & 300 & 1,5 & $9,16 \mathrm{E}-03$ & $8,85 \mathrm{E}-03$ & $8,74 \mathrm{E}-03$ & 1,20 \\
\hline 5 & 21,35 & 300 & 1,5 & $1,22 \mathrm{E}-02$ & $1,16 \mathrm{E}-02$ & 1,14E-02 & 1,52 \\
\hline 6 & 24,4 & 300 & 1,5 & $1,62 \mathrm{E}-02$ & $1,46 \mathrm{E}-02$ & $1,44 \mathrm{E}-02$ & 1,38 \\
\hline 7 & 27,45 & 300 & 1,5 & $1,80 \mathrm{E}-02$ & 1,79E-02 & 1,77E-02 & 1,34 \\
\hline 8 & 30,5 & 300 & 1,5 & $2,04 \mathrm{E}-02$ & $2,14 \mathrm{E}-02$ & $2,12 \mathrm{E}-02$ & 0,95 \\
\hline 9 & 21,35 & 300 & 1,5 & $4,44 \mathrm{E}-03$ & 1,16E-02 & 1,14E-02 & 1,52 \\
\hline 10 & 30,5 & 300 & 1,5 & $1,56 \mathrm{E}-02$ & $2,14 \mathrm{E}-02$ & 2,12E-02 & 0,95 \\
\hline 11 & 116 & 350 & 2,125 & 1,64E-01 & $1,72 \mathrm{E}-01$ & 1,71E-01 & 0,69 \\
\hline 12 & 141 & 350 & 2,875 & $1,75 \mathrm{E}-01$ & $2,18 \mathrm{E}-01$ & 2,16E-01 & 0,87 \\
\hline 13 & 107 & 350 & 2,875 & 1,21E-01 & $1,35 \mathrm{E}-01$ & 1,34E-01 & 0,70 \\
\hline 14 & 141 & 350 & 2,875 & $1,74 \mathrm{E}-01$ & $2,18 \mathrm{E}-01$ & 2,16E-01 & 0,87 \\
\hline 15 & 107 & 350 & 2,875 & 1,36E-01 & 1,35E-01 & 1,34E-01 & 0,70 \\
\hline 16 & 111 & 350 & 3,25 & $1,12 \mathrm{E}-01$ & 1,37E-01 & $1,36 \mathrm{E}-01$ & 0,94 \\
\hline 17 & 141 & 350 & 3,25 & 2,07E-01 & 2,07E-01 & $2,05 \mathrm{E}-01$ & 0,82 \\
\hline 18 & 141 & 350 & 3,25 & 1,91E-01 & 2,07E-01 & $2,05 \mathrm{E}-01$ & 0,82 \\
\hline 19 & 148 & 350 & 3,25 & $2,09 E-01$ & $2,25 E-01$ & $2,23 E-01$ & 0,78 \\
\hline 20 & 111 & 350 & 4,5 & $5,26 \mathrm{E}-02$ & 1,15E-01 & 1,14E-01 & 0,53 \\
\hline
\end{tabular}

Predicción de la susceptibilidad a la erosión (caso de estudio en un ambiente de campo)

En la Tabla 10 se muestran las predicciones de la metodología de cálculo en términos de velocidad límite erosiva, índice de desgaste erosivo, y tasa de erosión para cada una de las 23 líneas de flujo de los pozos correspondientes al caso de estudio en condiciones de campo. En primera instancia, se identifica que los sistemas que podrían ser susceptibles a erosión por una condición de alta velocidad de flujo son únicamente las líneas $\mathrm{CIC}$ 02, CIC-04, CIC-17 (se espera que exista erosión cuando la velocidad de flujo es casi igual o mayor a la velocidad límite erosiva predicha por la norma API RP 14E; véase índice desgaste erosión), de las cuales la línea $\mathrm{CIC}-17$ no presenta evidencia de desgaste por erosión.

Por otra parte, según la información experimental, se observa que 12 líneas de flujo (CIC-01 hasta CIC-12) muestran evidencia de erosión, de las 
cuales únicamente las líneas CIC-02 y CIC04 presentan información que coincide con los resultados predichos usando únicamente como criterio la velocidad de flujo. Por consiguiente, estos resultados muestran que la implementación de la norma API RP 14E tiene una eficiencia de predicción únicamente del $16,6 \%$ para los sistemas evaluados. Estos resultados se deben a que este modelo no considera el efecto de los sólidos, los cuales son un factor preponderante en el deterioro de la tubería, debido al impacto directo y repetitivo de las partículas sobre la superficie.

Con respecto al parámetro tasa de erosión, se observa que para la línea CIC-01, la metodología predice una tasa de erosión de 5,91 mpy, siendo categorizado como un sistema crítico por erosión según criterio establecido por Salama (tasa erosión $>4 \mathrm{mpy}$, desgaste inadmisible). Estos resultados son coherentes con la información experimental, debido a que esta línea de flujo ha presentado 2 fallas o rupturas (pérdida de contención del fluido) relacionadas con el mecanismo de daño de erosión durante su vida operativa. De manera que cuando se pronostica una tasa de erosión mayor a 4 mpy para un sistema en estudio, se recomienda implementar los programas de monitoreo de erosión, monitoreo de sólidos e inspección para hacer seguimiento a los procesos erosivos, y determinar la magnitud del daño mecánico sobre la tubería. Adicionalmente, se identifica que para las líneas de flujo donde se evidencia el efecto de la erosión en los cupones, la metodología predice tasas de erosión entre 5,66E-01 mpy y 2,1E-03 mpy. Finalmente, este análisis demuestra que existe concordancia entre los resultados obtenidos con el software CIC-Erosion y la información experimental de campo usada en el estudio.

Tabla 10. Resultados de erosión obtenidos con la implementación del software CIC-Erosion para los 23 sistemas de flujo

\begin{tabular}{|c|c|c|c|c|c|c|}
\hline $\begin{array}{l}\text { No. } \\
\text { Línea de } \\
\text { Flujo de } \\
\text { pozo }\end{array}$ & $\begin{array}{c}\text { Tasa } \\
\text { Producción } \\
\text { Arena (kg/s) }\end{array}$ & $\begin{array}{c}\text { Velocidad } \\
\text { de Flujo } \\
\text { (m/s) }\end{array}$ & $\begin{array}{l}\text { Velocidad } \\
\text { Límite } \\
\text { Erosiva } \\
\text { Predicha } \\
(\mathrm{m} / \mathrm{s})^{(\mathrm{A})}\end{array}$ & $\begin{array}{c}\text { Índice } \\
\text { Desgaste } \\
\text { Erosión (B) }\end{array}$ & $\begin{array}{c}\text { Tasa de } \\
\text { Erosión } \\
\text { Predicha } \\
\text { (mpy) }\end{array}$ & Información Experimental \\
\hline CIC-01 & 6,57E-03 & 2,97 & 6,90 & 0,43 & $5,91 \mathrm{E}+00$ & 2 Fallas, mecanismo de daño (Erosión) \\
\hline $\mathrm{CIC}-02$ & $6,17 \mathrm{E}-04$ & 3,91 & 3,99 & 0,98 & $5,66 \mathrm{E}-01$ & $\begin{array}{l}\text { Se evidencia desgaste en el cupón de } \\
\text { erosión }\end{array}$ \\
\hline $\mathrm{CIC}-03$ & $1,17 \mathrm{E}-03$ & 1,83 & 4,95 & 0,37 & $1,62 \mathrm{E}-01$ & Se evidencia Corrosión-Erosión \\
\hline CIC-04 & 2,73E-04 & 4,38 & 3,97 & 1,10 & $1,22 \mathrm{E}-01$ & Se evidencia Corrosión asistida por el flujo \\
\hline CIC-05 & $1,80 \mathrm{E}-03$ & 2,41 & 4,10 & 0,59 & $8,18 \mathrm{E}-02$ & Se evidencia Corrosión asistida por el flujo \\
\hline CIC-06 & $3,79 E-04$ & 2,16 & 4,44 & 0,49 & $5,24 \mathrm{E}-02$ & Se evidencia Corrosión asistida por el flujo \\
\hline CIC-07 & 2,55E-04 & 2,44 & 3,91 & 0,62 & 4,64E-02 & Se evidencia Corrosión asistida por el flujo \\
\hline CIC-08 & $1,04 \mathrm{E}-04$ & 2,97 & 3,90 & 0,76 & $3,56 \mathrm{E}-02$ & Se evidencia Corrosión asistida por el flujo \\
\hline CIC-09 & $3,00 E-04$ & 1,86 & 4,57 & 0,41 & $3,21 \mathrm{E}-02$ & Se evidencia Corrosión asistida por el flujo \\
\hline CIC-10 & $4,11 \mathrm{E}-05$ & 2,74 & 4,04 & 0,68 & 1,66E-02 & Se evidencia Corrosión asistida por el flujo \\
\hline CIC-11 & 3,08E-05 & 2,77 & 4,11 & 0,67 & $4,40 \mathrm{E}-03$ & Se evidencia Corrosión asistida por el flujo \\
\hline $\mathrm{ClC}-12$ & $6,08 \mathrm{E}-05$ & 1,81 & 3,93 & 0,46 & $2,10 \mathrm{E}-03$ & Se evidencia Corrosión asistida por el flujo \\
\hline CIC-13 & $3,78 \mathrm{E}-04$ & 0,94 & 3,96 & 0,24 & 1,35E-03 & Cupón de erosión en buen estado \\
\hline CIC-14 & $1,67 E-06$ & 2,73 & 5,11 & 0,54 & $8,35 \mathrm{E}-04$ & Cupón de erosión en buen estado \\
\hline CIC-15 & $1,04 \mathrm{E}-04$ & 1,24 & 4,01 & 0,31 & $7,83 \mathrm{E}-04$ & Cupón de erosión en buen estado \\
\hline CIC-16 & $6,75 \mathrm{E}-05$ & 1,01 & 3,93 & 0,26 & 2,83E-04 & Cupón de erosión en buen estado \\
\hline CIC-17 & $2,78 \mathrm{E}-07$ & 3,54 & 3,95 & 0,90 & 1,10E-04 & Cupón de erosión en buen estado \\
\hline CIC-18 & 3,53E-05 & 0,54 & 5,38 & 0,10 & $7,65 \mathrm{E}-05$ & Cupón de erosión en buen estado \\
\hline CIC-19 & $5,28 \mathrm{E}-06$ & 1,02 & 4,46 & 0,23 & 2,58E-05 & Cupón de erosión en buen estado \\
\hline CIC-20 & $4,42 \mathrm{E}-07$ & 2,28 & 4,75 & 0,48 & 2,11E-05 & Cupón de erosión en buen estado \\
\hline CIC-21 & $1,11 \mathrm{E}-06$ & 1,46 & 4,43 & 0,33 & 1,53E-05 & Cupón de erosión en buen estado \\
\hline CIC-22 & $3,98 E-06$ & 0,17 & 4,39 & 0,04 & $3,65 \mathrm{E}-08$ & Cupón de erosión en buen estado \\
\hline CIC-23 & $5,56 \mathrm{E}-10$ & 1,32 & 4,58 & 0,29 & 6,65E-09 & Cupón de erosión en buen estado \\
\hline
\end{tabular}

Notas: (A) Valor estimado aplicando la ecuación de la norma API RP14E, la cual no considera el efecto del contenido de sólidos, (B) Parámetro definido como la relación matemática (Velocidad de Flujo/Velocidad límite erosiva). 


\section{Conclusiones}

Con el fin de llevar la metodología de cálculo hasta un nivel operativo, se desarrolló una herramienta computacional de escritorio (CIC-Erosion) con la posibilidad de ser ejecutada en equipos de cómputo con sistemas operativos Windows 7 , 8 o 10. El enfoque y las directrices usadas en la fase de construcción permiten que la herramienta posea algunas ventajas, entre las cuales se destacan: a) acceso a la metodología general de cálculo de erosión; b) múltiples rutas de ingreso de información; c) información de algunos parámetros con el propósito de ayudar al usuario a establecer una magnitud veraz y confiable, y d) disponibilidad de un conversor de unidades para diferentes magnitudes físicas y químicas, y e) proporciona una experiencia de navegación clara y natural para el usuario del software.

A través de la fase de validación del funcionamiento operativo del software, se demostró que los resultados obtenidos con el software CIC-Erosion para cada uno de los modelos de predicción de tasa de erosión son totalmente veraces y confiables, y presentan los siguientes porcentajes de error: $0,0015 \%-0,00008 \%$ (modelo API RP 14E), 0,02 $\%-3 \%$ (modelo DNV RP 0501), 0,03 \% - 0,45 \% (modelo Salama), 0,25 \% (modelo Salama y Venkatesh), 0,53 \% - 1,57 \% (E/CRC Tulsa).

En la evaluación de la capacidad de predicción del software, se observó que existe concordancia entre los resultados obtenidos con la metodología de cálculo de erosión y la información experimental de campo usada en el estudio, ya que las mayores tasas de erosión predichas se presentan para los pozos que tienen evidencia de afectación por flujo o impacto de partículas sólidas en los cupones de erosión. De esta manera, la herramienta computacional CIC-Erosion puede ser considerada una alternativa eficiente $y$ confiable para la predicción del desgaste erosivo en infraestructura de sistemas de producción, recolección y transporte de hidrocarburos.

\section{Agradecimientos}

Los autores expresan su gratitud a la Corporación para la Investigación de la Corrosión $(\mathrm{CIC})$ por proporcionar los recursos necesarios para el desarrollo de esta investigación.

\section{Referencias bibliográficas}

[1] Schlumberger. Métodos prácticos de manejo de la producción de arena. Houston, Estados Unidos: 2004.

[2] Schlumberger. Recuperación del control de la producción de arena. Houston, Estados Unidos: 2007.

[3] American Petroleum Institute. API RP-14E Recommended practice for design and installation of offshore production platform piping systems. 5th Edition, Washington DC, USA: API, 1991.

[4] Salama M. An alternative to API 14E erosional velocity limits for sand-laden fluids. J. Energy Resour. Technol. ASME. 2000;122(2):71-7.

[5] McLaury BS, Shirazi SA, Rybicki EF. How operating and environmental conditions effect erosion. En: NACE International Annual Conference; 1999 Marzo; Houston, USA. Houston: 1999. Paper No 34.

[6] Salama MM, Venkatesh ES. Evaluation of API RP 14E erosional velocity limitations for offshore gas wells. En: 15th Annual Offshore Technology Conference; 1983 Mayo 2-5; Houston, USA. Houston: 1983. p. 371-6.

[7] Det Norske Veritas. DNV RP 0501 Recommended practice erosive wear in piping systems. Revisión 4.2, Noruega; DNV, 2007.

[8] Shirazi SA, Shadley JR, McLaury BS, Rybicki EF. A procedure to predict solid particle erosion in elbows and tees. J. Pressure Vessel Technol. 1995;117(1):45-52.

[9] McLaury BS, Shirazi SA. An alternate method to API RP 14E for predicting solids erosion in multiphase flow. J. Energy Resour. Technol. ASME. 2000;122(3):115-22.

[10] Mazumder QH, Shirazi SA, McLaury BS. A mechanistic model to predict sand erosion in multiphase flow in elbows downstrean of vertical pipes. En: NACE International Annual Conference; 2004 Marzo; Houston, USA. Houston: 2004. Paper No 04662.

[11] Mazumder QH. Prediction of erosion due to solid particle in single-phase and multiphase flows. J. Pressure Vessel Technol. ASME, 2007;129(4):576-82. 
[12] Mazumder QH, Shirazi SA, McLaury BS. Prediction of solid particle erosive wear of elbows in multiphase annular flow-model development and experimental validations. J. Energy Resour. Technol. ASME. 2008;130(2):023001-1-023001-10.

[13] Glaso O. Generalized pressure-volumetemperature correlations. JPT. 1980;32(5):78595.

[14] Beggs HD, Robinson JR. Estimating the viscosity of crude oil systems. JPT. 1975;27(9):1140-1.

[15] Standing MB. Volumetric and phase behavior of oil field hydrocarbon systems. USA: Millet the Printer, Inc.; 1977.

[16] Ahmed T. Reservoir engineering handbook. USA: Elsevier; 2010.

[17] Ghassan HA, Nimat B. Estimation of gas-oil surface tension. J. Pet. Sci. Eng. 2000;27(34):197-200.

[18] Meehan DN. A Correlation for water compressibility. Petroleum Engineer. 1980:125-126.

[19] American Petroleum Institute. API RP 581 Risk-Based Inspection Technology. 2th Edition,
Washington DC, USA: API, 2008.

[20] Sastri SRS, Rao KK. A simple method to predict surface tension of organic liquids. Chem. Eng. J.1995;59(2):181-6.

[21] Poling BE, Prausnitz JM. O'Connell JP. The properties of gases and liquids. USA: McGrawHill; 2004.

[22] Taitel Y, Dukler AE. A model for predicting flow regime transitions in horizontal and near horizontal gas-liqid flow. AIChE Journal. 1976;22(1):47-55.

[23] Ansari AM, Sylvester ND. A mechanistic model for upward bubble flow in pipes. AIChE J. 1988;34(8):1392-4.

[24] Jerez M, Jaramillo JE, Fuentes D. Prediction of multiphase flow in pipelines: literature Review. Ingeniería y Ciencia. 2015;11(22):213-33.

[25] Meng HC, Ludema KC. Wear models and predictive equations: their form and content. Wear. 1995;181-183:443-57.

[26] Parsi M, Najmi K, Najafifard F, Hassani S, McLaury B, Shirazi SA. A comprehensive review of solid particle erosion modeling of oil and gas wells and pipelines applications. J. Nat. Gas Sci. Eng. 2014;21:850-73. 